\title{
Topological automorphism groups of compact quantum groups
}

\author{
Alexandru Chirvasitu and Issan Patri
}

\begin{abstract}
We study the topological structure of the automorphism groups of compact quantum groups showing that, in parallel to a classical result due to Iwasawa, the connected component of identity of the automorphism group and of the "inner" automorphism group coincide.

For compact matrix quantum groups, which can be thought of as quantum analogues of compact Lie groups, we prove that the inner automorphism group is a compact Lie group and the outer automorphism group is discrete. Applications of this to the study of group actions on compact quantum groups are highlighted.

We end with the construction of a compact matrix quantum group whose fusion ring is not finitely generated, unlike the classical case.
\end{abstract}

Key words: automorphism group, outer automorphism group, fusion ring, compact quantum group, dynamical system

MSC 2010: 46L52; 46L55; 46L85; 16T05

\section{Introduction}

Compact quantum groups [34] as introduced in [33] (at the time under different terminology) are objects meant to capture the concept of "quantum symmetry" for various geometric or algebraic structures, and their construction in practice as quantum automorphism groups of the latter reflects this perspective.

This procedure has proven very flexible and fruitful, and the structures amenable to this type of treatment (and which hence give rise to interesting examples of compact quantum groups) include finite sets or possible non-commutative measure spaces [32], finite graphs [8], metric spaces [4, 11] or Riemannian manifolds $[6,12]$, to name only a few examples.

Actions of quantum groups on operator algebras (often equipped with various additional structures), as a generalization of actions of groups on operator algebras, can be regarded as noncommutative dynamical systems, with the dynamics in this case being encoded by the quantum group action. Here, we propose to study compact quantum groups themselves as dynamical objects, by investigating their own (classical) automorphism groups.

This line of inquiry continues the work of $[26,24]$ and in pursuing it, we have several aims.

First, these investigations fit well with the point of view, prevalent in the quantum group literature, that understanding the symmetries of a mathematical object (in our case a compact quantum group) sheds light on the underlying mathematical structure. For instance, the orbitboundedness-type result that constitutes Theorem 3.3 can be regarded as witnessing a type of "rigidity" for sufficiently small compact quantum groups (see also the discussion below, in the present section). Besides, a good understanding of the classical automorphism group of a compact quantum group would give insights towards understanding "quantum" automorphism groups of compact quantum groups. Attempts have been made towards defining such a notion [7, 20, 19]. 
Another source of motivation for us is the connection, in the classical case, between the inner / outer distinction for automorphisms of a compact group and the representation theory of the latter; see e.g. $[15,35]$ and references in the latter. Since compact quantum groups are essentially representation-theoretic objects (being defined, in one incarnation, as Hopf algebras that mimic algebras of matrix coefficients for finite-dimensional representations), this connection seems ripe for investigation in the quantum setting.

Finally, dynamics on compact groups is a rich topic in ergodic theory. Though we cannot possibly do it justice in this short introduction (see e.g. [14, 21, 23, 22, 17, 18, 27] and references cited there), we hope that the present work draws some analogies between that field and an incipient quantum analogue thereof by regarding compact quantum groups as non-commutative spaces being acted upon by structure-preserving transformations.

The paper is organized as follows.

In Section 1 we recall some of the background needed later, such as definitions, conventions, and so on.

Section 2 is devoted to the main results of the paper. We introduce for each compact quantum group $G$ its group of automorphisms $\operatorname{Aut}(G)$, and study the topology and structure of the latter. In first instance, we will see (Proposition 2.2, remark 2.3, and corollary 2.4) that there is essentially one reasonable way of topologizing $\operatorname{Aut}(G)$.

As for structure theory, we express $\operatorname{Aut}(G)$ as an extension of the outer automorphism group Out $(G)$ by the group $\operatorname{Inn}(G)$ of inner automorphisms and prove in Theorem 2.11 that the full automorphism group and the inner one share the same connected component of the identity. Moreover, Theorem 2.7 shows that for compact matrix quantum groups (typically regarded as noncommutative analogues of compact Lie groups) the outer automorphism group is discrete; this mimics the case of classical compact Lie groups.

In Section 3 we apply some of the contents of the preceding section to the study of dynamics on compact quantum groups, the main result (Theorem 3.3, paraphrased here) being that if a discrete group acts compactly on a compact matrix quantum group $G$, the resulting action on the dual object of irreducible representations of $G$ has uniformly bounded orbits. Next, in Theorem 3.4 using dynamical methods, we show that for a large family of compact matrix quantum groups, the outer automorphism group is finite and the automorphism group is a compact Lie group.

Finally, in Section 4 we present some examples of compact matrix quantum groups whose tensor categories of representations have infinitely-generated representation rings. This is in contrast to the classical case of compact Lie groups, for which it is well know that the representation rings are finitely generated [28, Corollary 3.3].

The relevance of this latter topic to the present work is that the question of whether or not such examples exist is motivated by the structure theory of the automorphism groups of compact matrix quantum groups (see Proposition 2.6).

\section{Acknowledgements}

This work was initiated at the 7th ECM satellite conference "Compact Quantum Groups" at Greifswald, Germany and the authors are grateful to the organizers, Uwe Franz, Malte Gerhold, Adam Skalski and Moritz Weber, for the invitation. The authors would also like to thank Yuki Arano and Makoto Yamashita for interesting discussions.

The first author was partially supported by NSF grant DMS - 1565226 . 


\section{Preliminaries}

In this section, we give a quick introduction to compact quantum groups and CQG algebras. We refer to [33], [34] and [9] for more details.

A compact quantum group $G=\left(A_{t}, \Phi\right)$ is a unital $C^{*}$-algebra $A_{t}$ together with a comultiplication $\Delta: A_{t} \rightarrow A_{t} \otimes A_{t}$ which is a coassociative $*$-homomorphism, such that the closure of the linear span of the sets $\left(A_{t} \otimes 1\right) \Delta\left(A_{t}\right)$ and $\left(1 \otimes A_{t}\right) \Delta\left(A_{t}\right)$ are dense in $A_{t} \otimes A_{t}$ in norm. Let $H$ be a finite dimensional Hilbert space with an orthonormal basis given by $\left\{e_{1}, e_{2}, \cdots, e_{n}\right\}$ and with $e_{i j}$, $1 \leqslant i, j \leqslant n$, the corresponding system of matrix units in $B(H)$. A unitary $u=\sum_{i, j} e_{i j} \otimes u_{i j} \in$ $B(H) \otimes A_{t}$ is said to be a finite dimensional representation of $G$, if $\Delta\left(u_{i j}\right)=\sum_{k} u_{i k} \otimes u_{k j}$ for all $1 \leqslant i, j \leqslant n$. Given two finite-dimensional representations $u \in B(H) \otimes A_{t}$ and $v \in B(K) \otimes A_{t}$, an operator $T \in B(H, K)$ is said to be an intertwiner of $u$ and $v$ if $(T \otimes i d) u=v(T \otimes i d)$. Two representations $u \in B(H) \otimes A_{t}$ and $v \in B(K) \otimes A_{t}$ are said to be equivalent if there exists an unitary intertwiner between them. A representation is said to be irreducible if the only self-intertwiners are the trivial ones. We denote the set of equivalence classes of irreducible representations of $G$ by $\operatorname{Irr}(G)$.

A compact matrix quantum group is a compact quantum group $G$ for which there exists a finite dimensional representation $u \in B(H) \otimes A_{t}$, with $u=\sum_{i, j} e_{i j} \otimes u_{i j}$, such that the elements $u_{i j}$ generate $A_{t}$ as a $C^{*}$-algebra. This is a generalization of the notion of compact lie groups (since for a compact lie group $G$, there always exists a faithful finite dimensional representation and thus, its coefficient functions generate $C(G)$ as a $C^{*}$-algebra).

Just like in the classical case of compact groups, for the case of a compact quantum group $G$ as well, there exists a canonical Hopf *-algebra $A$ which is a dense subalgebra of $A_{t}$ and is spanned by the matrix coefficients of the irreducible representations of $G$. This Hopf $*$-algebra is referred

to as the CQG algebra associated to $G$. If $G$ is a compact matrix quantum group, we will refer to $A$ as a CMQG algebra. It is also possible to traverse the converse route, whence starting with a CQG algebra $A$, which is defined as a Hopf algebra with some additional properties, one can then go on to associate a compact quantum group $G$ to this algebra [9].

We now consider an example, which will be important for us in the sequel. Let $n \geqslant 2$ be a positive integer, and recall the Hopf $*$-algebra $A_{u}(n)$ that is the function algebra of the so-called free unitary group $U_{n}^{+}$, as introduced in [30] (and in more general form in [29]). As a *-algebra, it is generated freely by $u_{i j}$ for $1 \leqslant i, j \leqslant n$ subject to the relations making

$$
\left(u_{i j}\right)_{i, j},\left(u_{i j}^{*}\right)_{i, j} \in M_{n}\left(A_{u}(n)\right)
$$

unitary.

The *-structures will not play a major role here, owing to the fact that Hopf subalgebras and quotient Hopf algebras of CQG algebras ([9]) are automatically CQG subalgebras and quotients respectively. In other words, so long as morphisms preserve the Hopf structures, the * structures come along for the ride.

\section{Automorphism groups of compact quantum groups}

In this section we study the topological structure of the automorphism group of compact quantum groups in general and of compact matrix quantum groups in particular. Before we move on to the main results of the section, let us recall some background on the topology of the automorphism group of a compact quantum group. 
Let $A$ be a CQG algebra underlying a compact quantum group $G$. Recall that $\operatorname{Aut}(G)$ (or Aut $(A))$ is defined in [26] as the group of coproduct-preserving automorphisms of the full enveloping $C^{*}$-algebra $\bar{A}$. Such automorphisms of compact quantum groups were first studied by Wang [31]. As noted there, restricting automorphisms to the dense Hopf *-subalgebra $A$ of $\bar{A}$ induces a bijection onto the group of Hopf *-algebra automorphisms of $A$. Moreover, this also bijects onto the group of coproduct-preserving automorphisms of the reduced $C^{*}$ envelope $\bar{A}_{r}$ of $A$ (see discussion preceding [26, Lemma 3.3]). We will henceforth freely identify these groups.

Recall furthermore that $\operatorname{Aut}(A)$ is topologized in [26] (discussion preceding Theorem 3.4) via the identity neighborhoods

$$
U\left(a_{1}, \cdots, a_{n} ; \varepsilon\right)=\left\{\alpha \in \operatorname{Aut}(A) \mid\left\|\alpha\left(a_{i}\right)-a_{i}\right\|<\varepsilon, \forall i\right\},
$$

where the norm used is that on $\bar{A}$.

Remark 2.1 This is initially defined for tuples $\left\{a_{i}\right\}$ in $\bar{A}$, but [26, Lemma 3.5] ensures it is enough to consider elements of the dense *-subalgebra $A$ of $\bar{A}$.

We will take here a slightly different approach to topologizing $\operatorname{Aut}(A)$, which will make it clear that in (2.1) we may as well have used the reduced $C^{*}$-norm of $\bar{A}_{r}$, or the $L^{2}$ norm associated to the Haar state, or the $L^{p}$ norm for some $p \geqslant 1$, etc. In other $\operatorname{words}$, $\operatorname{Aut}(G)$ is, not just as an abstract group, but even as a topological group, is determined by the quantum group structure of $G$, independent of the norm completion.

Consider an automorphism $\alpha$ of the Hopf $*$-algebra $A$. It will permute the simple subcoalgebras of $A$ and hence induce a permutation of the set $\operatorname{Irr}(G)$ of irreducible $A$-comodules. This provides a morphism

$$
\operatorname{Aut}(A) \rightarrow S_{\operatorname{Irr}(G)}
$$

where the latter symbol denotes the symmetric group on the set $\operatorname{Irr}(G)$. The kernel of this homomorphism consists of precisely those automorphisms of $A$ that preserve the class of each irreducible $\mathbb{G}$-representation, i.e. the normal subgroup

$$
\operatorname{Aut}_{\chi}(A) \preccurlyeq \operatorname{Aut}(A)
$$

of inner automorphisms of [26] (definition preceding Theorem 3.4). Note that every element of $\operatorname{Aut}_{\chi}(A)$ is uniquely determined by its effect on the simple subcoalgebras of $A$. Since the automorphism group of each such $n \times n$ matrix subcoalgebra is the projective $n \times n$ linear group, we have an embedding

$$
\operatorname{Aut}_{\chi}(A) \subset \prod_{V \in \operatorname{Irr}(G)} P G L(V) .
$$

All in all, we obtain an embedding

$$
\operatorname{Aut}(A) \subseteq\left(\prod_{V \in \operatorname{Irr}(G)} P G L(V)\right) \rtimes S_{\operatorname{Irr}(\mathrm{G})} .
$$

The right hand side of (2.2) has a natural topological group structure defined as the product topology on the set

$$
\left(\prod P G L(V)\right) \times S_{\operatorname{Irr}(G)},
$$


where the projective general linear groups have their standard Lie group topologies and the symmetric group $S_{\operatorname{Irr}(G)}$ is topologized via the compact-open topology resulting from the discrete topological space structure of $\operatorname{Irr}(G)$ (this, for instance, is the topology on a possibly infinite symmetric group used in $[5, \S 4])$.

The following result ensures that the topology on $\operatorname{Aut}(A)$ defined via the neighborhoods (2.1) is unambiguous with respect to the norm used therein by identifying it with the topology inherited from the embedding (2.2).

Proposition 2.2 Let $A$ be a $C Q G$ algebra, and $\|\cdot\|$ a norm on A making the latter into a normed complex vector space such that the Haar state $h: A \rightarrow \mathbb{C}$ is continuous. Then, the topology on $\operatorname{Aut}(A)$ defined via the identity neighborhoods

$$
U\left(a_{1}, \cdots, a_{n} ; \varepsilon\right)=\left\{\alpha \in \operatorname{Aut}(A) \mid\left\|\alpha\left(a_{i}\right)-a_{i}\right\|<\varepsilon,\left\|\alpha^{-1}\left(a_{i}\right)-a_{i}\right\|<\varepsilon, \forall a_{i}\right\}
$$

for various tuples $\left\{a_{i}\right\} \subset A$ and $\varepsilon>0$ coincides with the subspace topology inherited from (2.2).

Proof Let us denote the topology coming from (2.2) by TOP ${ }_{e m b}$ (for embedding) and the topology defined via $(2.3)$ by $\mathrm{TOP}_{\|\cdot\|}$. We want to argue that each contains the other. Since both make $\operatorname{Aut}(A)$ into a topological group, this is equivalent to proving that open identity neighborhoods with respect to each topology contain open identity neighborhoods with respect to the other; we will do this separately for the two inclusions.

(1): $\mathrm{TOP}_{e m b} \subseteq \mathrm{TOP}_{\|\cdot\|}$. Let $U \subseteq \operatorname{Aut}(A)$ be an identity neighborhood in $\mathrm{TOP}_{e m b}$. Fix a simple $A$-comodule $V$, and denote by $C \subset A$ its coefficient coalgebra. Then we may as well assume $U$ is of the form

$$
U=\left\{\alpha \in \operatorname{Aut}(A)|\alpha(C)=C, \alpha|_{C} \in \mathcal{U} \subset P G L(V) \cong \operatorname{Aut}(C)\right\}
$$

where $\mathcal{U}$ is a neighborhood of the identity in the Lie group $P G L(V)$. Indeed, intersections of such open identity neighborhoods in $\mathrm{TOP}_{e m b}$ form a basis (around $1 \in \operatorname{Aut}(A)$ ) for the latter topology.

Let $\chi_{V} \in C$ be the character of $V$. Then we claim that if $\varepsilon>0$ is small enough, then $U\left(\chi_{V} ; \varepsilon\right)$ (defined as in (2.3)) consists of elements $\alpha \in \operatorname{Aut}(A)$ with $\alpha(C)=C$ (or equivalently, $\left.\alpha\left(\chi_{V}\right)=\chi_{V}\right)$. Indeed, $\alpha\left(\chi_{V}\right)$ is a character $\chi_{W}$ for some irreducible $A$-comodule $W$, and the conclusion that $W \cong V$ for sufficiently small $\varepsilon$ follows from the continuity of $h$ and the fact that

$$
h\left(\chi_{V} \chi_{W}^{*}\right) \in\{0,1\},
$$

with 1 if $W \cong V$ and 0 otherwise.

Finally, having chosen $\varepsilon$ small enough to ensure that for any $\alpha \in U\left(\chi_{V} ; \varepsilon\right), \alpha(C)=C$, we can shrink it further to make sure that for a basis $u_{i j}, 1 \leqslant i, j \leqslant n$ of $C$ the restriction of every

$$
\alpha \in U\left(u_{i j}, 1 \leqslant i, j \leqslant n ; \varepsilon\right)
$$

to $C$ is contained in the neighborhood $\mathcal{U} \subset P G L(V)$. Indeed, this follows from the fact that since $C$ is finite-dimensional, all topologies making it into a complex topological vector space coincide.

(2): $\mathrm{TOP}_{\|\cdot\|} \subseteq \mathrm{TOP}_{e m b}$. It is sufficient to select an open set of the form (2.3) with $n=1$, and $a=a_{1} \in A$ belonging to some simple subcoalgebra $C \subset A$. Clearly then, for a small enough neighborhood $\mathcal{U}$ of the identity in $P G L(C)$, the set

$$
\left\{\alpha \in \operatorname{Aut}(A)|\alpha(C)=C, \alpha|_{C} \in \mathcal{U} \subset P G L(V) \cong \operatorname{Aut}(C)\right\}
$$


used in the proof of part (1) will be contained in $U(a ; \varepsilon)$.

This finishes the proof of part (2) and of the proposition.

Remark 2.3 The proof of Proposition 2.2 given above extends to prove a stronger statement applying to topologies on $\operatorname{Aut}(A)$ defined analogously to (2.3), via neighborhoods of the form

$$
U\left(a_{i} ; \mathcal{U}\right)=\left\{\alpha \in \operatorname{Aut}(A) \mid \alpha\left(a_{i}\right)-a_{i} \in \mathcal{U} \ni \alpha^{-1}\left(a_{i}\right)-a_{i}, \forall i\right\}
$$

where $\mathcal{U}$ is a neighborhood of $0 \in A$ with respect to some topological vector space structure on $A$ making $h: A \rightarrow \mathbb{C}$ continuous.

We now record a consequence of Proposition 2.2. As noted above, given a compact quantum group we can also define the group $\operatorname{Aut}_{r}(A)$ of coproduct-preserving automorphisms of the reduced $C^{*}$-algebra $\bar{A}_{r}$. We understand $\operatorname{Aut}_{r}(A)$ to be topologized by the identity neighborhoods (2.3) for the reduced $C^{*}$-norm $\|\cdot\|$.

Since any $\alpha \in \operatorname{Aut}(A)$ preserves the Haar state, it defines a unique element in $\operatorname{Aut}_{r}(A)$. Hence, we have a canonical map $\operatorname{Aut}(A) \rightarrow \operatorname{Aut}_{r}(A)$ which is clearly a group homomorphism. It also easy to show that this map is bijective. The inverse bijection is constructed in the following way. Since any $\alpha \in \operatorname{Aut}_{r}(A)$ restrict to an automorphism of the dense Hopf $*$-algebra $A$, it extends uniquely by the universal property to an automorphism in $\operatorname{Aut}(A)$.

Similarly, since any automorphism of $\bar{A}_{r}$ intertwining $\Delta$ has a unique normal extension to $\mathrm{L}^{\infty}(G)$, it induces a bijective map $\operatorname{Aut}_{r}(A) \rightarrow \operatorname{Aut}_{\infty}(A)$, where the latter is the set of comultiplicationpreserving automorphisms of the reduced von Neumann algebra $L^{\infty}(G)$. We topologize $\operatorname{Aut}_{\infty}(A)$ by neighborhoods analogous to (2.3).

Now, Proposition 2.2 proves

Corollary 2.4 For any compact quantum group $G$ with underlying $C Q G$ algebra $A$ the groups $\operatorname{Aut}(A) \operatorname{Aut}_{r}(A)$ and $\operatorname{Aut}_{\infty}(A)$ are isomorphic as topological groups.

As mentioned above, the problem of seeking CMQGs whose fusion algebra is not finitely generated was prompted by the question of whether or not the outer automorphism group Out $(A)$ of a CMQG algebra $A$ is discrete. In fact, for a CMQG $G$, if the fusion ring $Z(G)$ is finitely generated, then the group $\operatorname{Out}(G)$ is discrete. This is not hard to see and was exhibited in [24]. We recap the proof here for the convenience of the reader.

Lemma 2.5 Let $[u] \in \operatorname{Irr}(G)$ and let $\chi(u)$ denote the character of $u$. The stabilizer subgroup of $u$ defined by

$$
K_{u}:=\{\alpha \in \operatorname{Aut}(\Gamma): \alpha(\chi(u))=\chi(u)\}
$$

is an open subgroup of $\operatorname{Aut}(G)$.

Proof Consider the open set defined by $V(\chi(u), 1)=\{\alpha \in \operatorname{Aut}(G):\|\chi(u)-\alpha(\chi(u))\|<1\}$. Note that $h\left(\chi(u)^{*} \chi(u)\right)=1$, and for $\alpha \in \operatorname{Aut}(G)$ one has $h\left(\alpha(\chi(u))^{*} \chi(u)\right)=1$ if $\alpha \in K_{u}$ and zero otherwise. So, for $\alpha \in V(\chi(u), 1)$, we have

$$
\left|h\left(\chi(u)^{*} \chi(u)\right)-h\left(\alpha(\chi(u))^{*} \chi(u)\right)\right| \leqslant\|\chi(u)-\alpha(\chi(u))\|<1
$$

and hence $\left|1-h\left(\alpha(\chi(u))^{*} \chi(u)\right)\right|<1$. It follows that $\alpha \in K_{u}$. Consequently, $K_{u}$ is indeed an open subgroup of $\operatorname{Aut}(G)$.

Using the above lemma, it was shown in [26] that the group Out $(G)$ for any compact quantum group $G$ is totally disconnected. 
Proposition 2.6 Suppose the fusion ring $Z(G)$ of $G$ is finitely generated as a ring. Then Out $(G)$ is discrete, equivalently $\operatorname{Aut}_{\chi}(G)$ is an open subgroup of $\operatorname{Aut}(G)$.

Proof Denote the generators of $Z(G)$ by $\lambda_{1}, \lambda_{2}, \cdots, \lambda_{n}$. The characters of irreducible representations of $G$ form a basis of $Z(G)$. Define the set

$$
N:=\left\{\chi(u): \chi(u) \text { appears in the linear decomposition of some } \lambda_{i}, 1 \leqslant i \leqslant n\right\},
$$

where $[u] \in \operatorname{Irr}(G)$. Clearly, $N \subseteq G_{\text {char }}$ is finite, where $G_{\text {char }}$ deotes the set of characters of $G$. Note that $\alpha \in \operatorname{Aut}(G)$ is in $\operatorname{Aut}_{\chi}(G)$ if and only if $\alpha(\chi(u))=\chi(u)$ for all $\chi(u) \in N$. Indeed, observe that $\alpha\left(\lambda_{i}\right)=\lambda_{i}$ for all $1 \leqslant i \leqslant n$ if $\alpha(\chi(u))=\chi(u)$ for all $\chi(u) \in N$. But since $\lambda_{i}$ generate $Z(G)$ (as a ring), we have $\alpha(a)=a$ for all $a \in Z(G)$ if $\alpha(\chi(u))=\chi(u)$ for all $\chi(u) \in N$. Since $G_{c h a r} \subseteq Z(G)$ it follows that $\alpha \in A_{u}(G)$. The other implication is obvious.

To complete the proof, fix $[u] \in \operatorname{Irr}(G)$ and consider the stabilizer group $K_{u}$ defined in Lemma 2.5. Write $\mathcal{O}=\{[u] \in \operatorname{Irr}(G): \chi(u) \in N\}$. Observe that $\operatorname{Aut}_{\chi}(G)=\cap_{[u] \in \mathcal{O}} K_{u}$. Since $N$ is finite use Lemma 2.5 to finish the proof.

Thus, finite generation of the fusion ring for a compact matrix quantum group would have provided an affirmative answer to the question whether its outer automorphism group is discrete, which is true in the classical case of compact Lie groups, hence the relevance of that problem. However, we show that there exist examples of compact matrix quantum for which fusion ring is not finitely generated as a ring. However, inspite of such examples (see Section 4 below), we can show that the outer automorphism group of a $C M Q G$ is indeed discrete. This illustrates an interesting phenomena in the theory of quantum groups, where the examples are diverse enough for such examples to exist, but at the same time, the theory is nice enough to allow us to prove unified results for all quantum groups.

Theorem 2.7 For any $C M Q G$ A the group $\operatorname{Out}(A)$ is discrete.

Proof Let

$$
C=\operatorname{span}\left\{u_{i j}, 1 \leqslant i, j \leqslant n\right\}
$$

be a subcoalgebra generating $A$ as an algebra, and corresponding to some $A$-comodule $U$ with basis $e_{i}$, with coaction

$$
e_{j} \mapsto \sum_{i} e_{i} \otimes u_{i j}
$$

We will show that the group $\operatorname{Aut}_{\chi}(A)$ of inner automorphisms of $A$ is open. Equivalently, we have to show that if an automorphism $\alpha$ of $A$ is sufficiently close to the trivial automorphism $1 \in \operatorname{Aut}=\operatorname{Aut}(A)$ in the standard topology on Aut then it is inner.

Sufficiently close to 1 certainly implies that $\alpha$ preserves the class of $U$, and hence acts on the coalgebra $C$. This means in particular that some open neighborhood of $1 \in$ Aut is contained in the isotropy group $\mathrm{Aut}_{U}$ of (the class of) $U$.

Every element in Aut $_{U}$ acts on the coalgebra $C$, and moreover, since the latter generates $A$ as an algebra, we have an embedding $\operatorname{Aut}_{U} \rightarrow \operatorname{Aut}(C)$. The latter is a closed Lie subgroup of $P G L_{n}(\mathbb{C})$ (the latter being the automorphism group of an $n \times n$ matrix coalgebra), and moreover the inclusion

$$
\operatorname{Aut}_{U} \subseteq \operatorname{Aut}(C) \subseteq P G L_{n}(\mathbb{C})
$$


is easily seen to be closed (see Lemma 2.8 below). In conclusion $\mathrm{Aut}_{U}$ is a Lie group. But this then implies that some neighborhood $\mathcal{V}$ of the identity in $\mathrm{Aut}_{U}$ (and hence also in Aut) is a (path)connected Lie group.

Now consider the action of elements $\beta$ in the neighborhood $\mathcal{V}$ on some fixed simple subcoalgebra $D \subset A$. The image $\beta(D)$ is a simple subcoalgebra of $A$ that either coincides with $D$, or is orthogonal to $D$ with respect to the Haar state. The fact that $1 \in$ Aut fixes $D$ and can be connected to $\beta$ by a path rules out the latter possibility, and hence $\beta(D)=D$.

It follows from the previous paragraph that $\beta$ fixes the class of the irreducible comodule corresponding to $D$; since this comodule was chosen arbitrarily from among the irreducible representations of the quantum group, we obtain the desired conclusion that $\beta$ acts trivially on the representation ring of $A$.

Lemma 2.8 Let $A$ be a normed complex algebra and $C \leqslant A$ a finite-dimensional linear subspace. Then, the embedding

$$
\operatorname{Aut}(A, C) \subseteq P G L(C)
$$

is closed.

Proof The elements of $\operatorname{Aut}(C)$ that arise by restriction from $\operatorname{Aut}(A, C)$ are precisely those that preserve all relations between elements of $C$. But the fact that $A$ is a topological algebra implies that the preservation of each relation is a closed condition, and the conclusion follows.

Corollary 2.9 For any compact matrix quantum group $G$, the automorphism group $\operatorname{Aut}_{\chi}(G)$ is a compact Lie group.

Proof First let us note that $\operatorname{Aut}_{\chi}(G)$ is compact (see Theorem 3.4 of [26]). Now, as shown in the proof of Theorem 2.7, the group Aut $U$ is a Lie group. It is easy to see that $\operatorname{Aut}_{\chi}(G)$ is a closed subgroup of $\mathrm{Aut}_{U}$ and hence is itself a Lie group.

Corollary 2.10 For any compact matrix quantum group $G$, the automorphism group $\operatorname{Aut}(G)$ is a Lie group.

Proof For a compact matrix quantum group $G$, the normal subgroup $\operatorname{Aut}_{\chi}(G) \preccurlyeq \operatorname{Aut}(G)$ (which is compact and Lie according to Corollary 2.9) is open by Theorem 2.7. In conclusion $\operatorname{Aut}(G)$ is an extension of a discrete group by a Lie group, and is hence Lie.

We now proceed to prove an Iwasawa-type theorem for quantum groups. One of the main results of the seminal paper [16] states that for a compact group $G$, the connected component of the identity (henceforth connected component) of the automorphism group of $G$ equals the connected component of the inner automorphism group of $G$.

Let now $G$ be a compact quantum group. Let us denote the connected component of Aut $(G)$ by $\operatorname{Aut}^{0}(G)$ and the connected component of the group $\operatorname{Aut}_{\chi}(G)$ by $\operatorname{Aut}_{\chi}^{0}(G)$.

Theorem 2.11 For any compact quantum group $G$, we have $\operatorname{Aut}^{0}(G) \cong \operatorname{Aut}_{\chi}^{0}(G)$.

Proof It is obvious that $\operatorname{Aut}_{\chi}^{0}(G) \subseteq \operatorname{Aut}^{0}(G)$. So, the theorem will follow if we can show that if $\alpha \in \operatorname{Aut}^{0}(G)$, then $\alpha \in \operatorname{Aut}_{\chi}^{0}(G)$. To this end, we fix an irreducible representation $u^{a}$ of $G$, with character denoted by $\chi_{a}$ and define the following continuous function

$$
f_{a}: \operatorname{Aut}(G) \rightarrow\{0,1\}
$$




$$
\alpha \mapsto h_{G}\left(\chi_{a}^{*} \alpha\left(\chi_{a}\right)\right)
$$

It is easy to see that $f_{a}$ is a continuous function. Now, when $\alpha$ is the trivial automorphism, then we have that $f_{a}(\alpha)=1$, hence, we have, by connectedness of $\operatorname{Aut}^{0}(G)$, that for any $\alpha \in \operatorname{Aut}^{0}(G)$, $f_{a}(\alpha)=1$, which implies that $\alpha\left(\chi_{a}\right)=\chi_{a}$. Since, the choice of the irreducible representation was arbitrary, we have $\alpha \in \operatorname{Aut}_{\chi}(G)$, and hence, the result follows.

This then allows us to prove a straightforward corollary.

Corollary 2.12 Let $G$ be a compact quantum group. Then the connected component of the group $\operatorname{Aut}(G)$ is a compact group. If $G$ is a compact matrix quantum group, then the connected component of $\operatorname{Aut}(G)$ is a compact Lie group.

Proof This follows easily from the previous theorem and from the compactness of the group $\operatorname{Aut}_{\chi}(G)$ (see Theorem 3.4 of [26]). In the compact matrix quantum group case, this follows from the previous theorem and Corollary 2.9 .

In fact, one can prove stronger structural results about the group $\operatorname{Aut}(G)$ if a little more is known about the compact quantum group $G$.

Proposition 2.13 Let $G$ be a compact matrix quantum group such that $\operatorname{Out}(G)$ is finite. Then $\operatorname{Aut}(G)$ is compact Lie group.

Proof Since Out $(G)=\operatorname{Aut}(G) / \operatorname{Aut}_{\chi}(G)$, and since $\operatorname{Aut}_{\chi}(G)$ is a compact Lie group in case that $G$ is a compact matrix quantum group, hence, when $\operatorname{Out}(G)$ is finite, it follows easily that $\operatorname{Aut}(G)$ is a compact Lie group.

Corollary 2.14 Let $G$ be a compact quantum group with fusion rules identical to that of simple compact Lie group. Then $\operatorname{Aut}(G)$ is a compact Lie group.

Proof This follows from the previous proposition and from the fact that for a compact quantum group $G$ with fusion rules isomorphic to that of a simple compact Lie group, the group Out $(G)$ can only have order $1,2,3$ or 6 (see Proposition 3.8 of [26]).

In order to state the following auxiliary result that has come up in the above proof, we place ourselves in the following context: $A$ is a topological complex algebra, $C \leqslant A$ is a finite-dimensional linear subspace that generates $A$, and $\operatorname{Aut}(A, C)$ is the group of automorphisms of $A$ that leave $C$ invariant.

Under the above setup, restricting elements of $\operatorname{Aut}(A, C)$ to $C$ gives rise to an inclusion

$$
\operatorname{Aut}(A, C) \subseteq \operatorname{Aut}(C) \cong P G L(C)
$$

As before, we topologize the group $\operatorname{Aut}=\operatorname{Aut}(A)$ pointwisely using the topology of $A$ : the neighborhoods of $1 \in$ Aut are given by

$$
\left\{g \in \text { Aut } \mid g a_{i}-a_{i}, g^{-1} a_{i}-a_{i} \in U, \forall i\right\}
$$

for various choices of neighborhoods $U \subseteq A$ of 0 and finite sets $\left\{a_{i}\right\} \subset A$.

Remark 2.15 We have included both $g$ and $g^{-1}$ in (2.4) in order to ensure that Aut is a topological group and hence the inverse is continuous.

The omission of $g^{-1}$ in the analogous definition from [26] (see the discussion preceding Theorem 3.4 therein) makes no difference, since in that case the action is by isometries with respect to a $C^{*}$ norm on $A$. 


\section{Some applications to CQG dynamical systems}

In this section we use the results of last section, to draw some quick conclusions about spectral properties of CQG dynamical systems in certain cases.

In the paper [24], the notion of CQG dynamical systems was introduced and studied, which is a tuple $(G, \Gamma, \alpha)$, where $G$ is a compact quantum group and $\Gamma$ acts on $C(G)$ by quantum group automorphisms, with $\alpha$ denoting the action. Thus, we have a group homomorphism $\alpha: \Gamma \rightarrow$ $\operatorname{Aut}(G)$.

Definition 3.1 Let $(G, \Gamma, \alpha)$ be a CQG dynamical system. Let $\|\cdot\|$ denote the $C^{*}$-norm on $A$.

1. [2] We say that the action is almost periodic if given any $a \in A$, the set $\left\{\alpha_{\gamma}(a): \gamma \in \Gamma\right\}$ is relatively compact in $A$ with respect to $\|\cdot\|$.

2. We say that the action is compact if given any $a \in A$, the set $\left\{\alpha_{\gamma}(a) \Omega_{h}: \gamma \in \Gamma\right\}$ is relatively compact in $L^{2}(A)$ with respect to the $\|\cdot\|_{2, h}$.

3. The extended action of $\Gamma$ on $L^{\infty}(G)$ is compact if given any $a \in L^{\infty}(G)$, the set $\left\{\alpha_{\gamma}(a) \Omega_{h}\right.$ : $\gamma \in \Gamma\}$ is relatively compact in $L^{2}(A)$ with respect to the $\|\cdot\|_{2, h}$.

The following theorem was proved in [24] (see Theorem 3.9)

Theorem 3.2 Let $(G, \Gamma, \alpha)$ be a $C Q G$ dynamical system. TFAE:

(i) The action is almost periodic;

(ii) the action is compact;

(iii) the orbit of any irreducible representation in $\operatorname{Irr}(G)$ is finite;

(iv) the extended action of $\Gamma$ on $L^{\infty}(G)$ is compact.

It then follows immediately that if the action is "inner" in the sense that $\alpha(\Gamma) \subset \operatorname{Aut}_{\chi}(G)$, then the corresponding CQG dynamical system $(G, \Gamma, \alpha)$ is compact. This is because it follows from the definition of $\operatorname{Aut}_{\chi}(G)$ that the induced action of $\Gamma$ on $\operatorname{Irr}(G)$ is trivial. In this context, a pertinent question to ask is whether one can obtain a result converse to this. In other words, suppose we have a compact CQG dynamical system, to what extent is that action "inner". It was observed in [24], that for a compact quantum group $G$, with $\operatorname{Out}(G)$ discrete, any compact CQG dynamical system is virtually inner. Thus, in case $G$ is a compact matrix quantum group, we have the following

Theorem 3.3 Let $G$ be a compact matrix quantum group. Let $(G, \Gamma, \alpha)$ be a compact $C Q G d y$ namical system. Then the subgroup

$$
\Gamma_{\chi}:=\left\{\gamma \in \Gamma: \alpha_{\gamma} \in \operatorname{Aut}_{\chi}(G)\right\}
$$

of $\Gamma$ is of finite index.

Proof Recall that any $C^{*}$-dynamical system $(A, \Gamma, \alpha)$ is almost periodic if and only if the closure of the image of $\Gamma$ under $\alpha$ in $\operatorname{Aut}(A)$ is compact in the pointwise norm topology (see Theorem 2.2 and Corollary 2.7 of [13]). Thus, since $(G, \Gamma, \alpha)$ is compact, it follows that $H:=\bar{\Gamma} \subseteq \operatorname{Aut}(G) \subseteq \operatorname{Aut}(A)$ is compact. But we have, by Theorem $2.7 \operatorname{Out}_{\chi}(G)$ is $\operatorname{discrete}$ equivalently $\operatorname{Aut}_{\chi}(G)$ is an open subgroup of $\operatorname{Aut}(G)$. So, $H_{\chi}=\operatorname{Aut}_{\chi}(G) \cap H$ is open in $H$. Since $H$ is compact, so $H_{\chi}$ is of finite index in $H$. Consequently, $\Gamma_{\chi}$ is finite index subgroup of $\Gamma$.

The next result uses the ideas of CQG dynamical systems to give a structural result on automorphism groups. 
Theorem 3.4 Suppose that $G$ is a compact quantum group and suppose that $u=\left(\left(u_{i j}\right)\right)$ is a $m$-dimensional representation of $G$ such that the $C^{*}$-algebra generated by $u_{i j}, i, j \in\{1,2, \ldots, m\}$ in $C_{m}(G)$ is itself $C_{m}(G)$. Suppose further that up to unitary equivalence, there are only finitely many $n$-dimensional representations of $G$. Then, $\operatorname{Aut}(G)$ and $\operatorname{Aut}_{\chi}(G)$ are compact Lie groups and $\operatorname{Out}(G)$ is a finite group.

Proof Since $G$ is a compact matrix quantum group, it follows that $C_{m}(G)$ is separable, and therefore, $\operatorname{Aut}\left(C_{m}(G)\right)$ is separable (in fact is Polish). It follows that $\operatorname{Aut}(G)$ is separable. Let now $\Gamma$ be a dense countable subgroup of $\operatorname{Aut}(G)$. Hence, we have a CQG dynamical system $(G, \Gamma)$. It follows then from the hypothesis that under the induced $\Gamma$-action on $\operatorname{Irr}(G)$, the orbit $\{\Gamma[u]\}$ is finite (here $[u]$ denotes the equivalence class corresponding to the representation $u$ of $G$ ). Applying Proposition 6.7 of [24], we get then that the orbit of any element of $\operatorname{Irr}(G)$ under the induced $\Gamma$-action is finite, whence it follows, using Theorem 3.2, that the CQG dynamical system $(G, \Gamma)$ is compact.

However, since $\Gamma$ is dense in $\operatorname{Aut}(G)$, we then have that $\operatorname{Aut}(G)$ is compact, using Theorem 2.2 and Corollary 2.7 of [13]. But, by Theorem 2.7, Out $(G)$ is discrete for $G$, and since Aut $(G)$ is compact, we have that $\operatorname{Out}(G)$ is finite. Now, by Corollary 2.9, $\operatorname{Aut}_{\chi}(G)$ is a compact Lie group and so, finiteness of $\operatorname{Out}(G)$ implies that $\operatorname{Aut}(G)$ is also a compact Lie group. Hence, we are done.

Remark 3.5 Let us note that the condition mentioned above, of compact matrix quantum groups whose generating representation has some fixed dimension, say $m$, and there are only finitely many $m$-dimensional representations up to unitary equivalence, is satisfied by several examples, including the free unitary quantum groups, the free orthogonal quantum groups, the free permutation quantum groups, $q$-deformations of simple compact Lie groups, Rieffel deformations of simple compact Lie groups, etc.

\section{CMQGs with infinitely generated fusion rings}

In this section we construct a compact matrix quantum group whose complex fusion algebra is not finitely generated as an algebra. In fact, the CQG algebra we consider is not only finitely generated (hence corresponding to a CMQG), but even finitely presented.

Throughout the rest of this section $n \geqslant 2$ is understood to be an arbitrary but fixed positive integer, and we denote $A=A_{u}(n)$. The CQG algebra $C$ that will provide the counterexample fits into an exact sequence

$$
\mathbb{C} \longrightarrow C(\mathbb{Z} / 2) \longrightarrow C \longrightarrow \mathbb{C}
$$

of Hopf algebras in the sense of [1]. The construction is also a generalization of that of a bicrossed product as in [10], where the compact group is $\mathbb{Z} / 2$, and the discrete group is the quantum discrete dual to the free unitary group $U_{n}^{+}$(i.e. the discrete quantum group with group algebra $A$ ).

We construct $C$ as a bicrossed product $C(\mathbb{Z} / 2) \sharp A$ according to the notation of [1], following the recipe outlined in [1, Theorem 2.20]. According to (a very particular version of) that result, all we need for such a construction to go through is a coaction

$$
A \rightarrow A \otimes C(\mathbb{Z} / 2)
$$

(i.e. an action of the group $\mathbb{Z} / 2$ on $A$ ) satisfying certain conditions. Unpacking that theorem, it follows that the construction does indeed result in a Hopf algebra structure on $C=C(\mathbb{Z} / 2) \otimes A$ if 
- $\mathbb{Z} / 2$ acts on $A$ by Hopf algebra automorphisms;

- $C$ is equipped with the tensor product algebra structure;

- $C$ is equipped with the smash coproduct [1, (2.15)].

In reference to the third bullet point, we apply [1, (2.15)] by taking $\rho: A \rightarrow A \otimes C(\mathbb{Z} / 2)$ to be our coaction defining the $(\mathbb{Z} / 2)$-action and $\tau: A \rightarrow \mathbb{C}(\mathbb{Z} / 2)^{\otimes 2}$ to be the trivial "cycle"

$$
A \ni a \mapsto \varepsilon(a)(1 \otimes 1) \in C(\mathbb{Z} / 2)^{\otimes 2} .
$$

Moreover, (4.1) is a central extension, in the sense that $C(\mathbb{Z} / 2)$ is contained in the center of $C$.

The action of $\mathbb{Z} / 2=\langle\gamma\rangle$ on $A$ will be defined as

$$
\gamma: u_{i j} \leftrightarrow u_{i j}^{*}
$$

at the level of generators, and extended in the only way possible to a $*$-algebra automorphism (the relations of $A$ ensure that this is indeed possible). The $*$-structure on $C$ is simply the tensor product $*$-structure on $C(\mathbb{Z} / 2) \otimes A$, and is easily seen to make $C$ into a CQG algebra in the sense of $[9]$.

We now need to understand the fusion ring $R(C)$ of $C$, in order to show that it is not finitely generated. In fact, we will prove this for the complexified version of $R$, namely

$$
R_{\mathbb{C}}=R_{\mathbb{C}}(C)=\mathbb{C} \otimes_{\mathbb{Z}} R(C)
$$

(the fact that $R_{\mathbb{C}}$ is not finitely generated as a complex algebra is a priori stronger than $R(C)$ not being finitely generated as a ring).

Proposition 4.1 below describes the comodule category of $C$, achieving the above-stated goal. Before giving the statement we recall various results on the representation theory of $U_{n}^{+}$from [3] (see Théorème 1 therein) and make a few preparatory remarks.

The standard $A$-comodule $V$ whose underlying matrix coalgebra is spanned by the generators $u_{i j}$ and its dual $V^{*}$ (with matrix coalgebra spanned by $u_{i j}^{*}$ ) generate the fusion ring of $U_{n}^{+}$. The irreducible representations of $U_{n}^{+}$are in bijection with the words on the alphabet

$$
\alpha=[V], \beta=\left[V^{*}\right]
$$

(classes in the fusion semiring $R_{+}(A)$ ), in the sense that for every word the corresponding tensor product of copies of $V$ and $V^{*}$ has a unique simple summand that does not appear as a summand in a strictly shorter word.

One consequence of this is that the fusion ring $R(A)$ is free (as a ring) on the generators $\alpha$ and $\beta$. Indeed, the description of the fusion rules in [3, Théorème 1 (i)] makes it clear that $R(A)$ admits a filtration by degree with

$$
\operatorname{deg}(\alpha)=1=\operatorname{deg}(\beta)
$$

such that the associated graded ring is indeed free, and hence the canonical map from the free ring on two generators sending these generators to $\alpha$ and $\beta$ respectively is an isomorphism of rings.

Now, the automorphism $\gamma$ defined as in (4.2) implements an action by monoidal autoequivalences on the category $\mathcal{M}=\mathcal{M}^{A}$ of finite-dimensional comodules by twisting via the automorphism $\gamma$. We denote this twisting functor by $\gamma^{*}$. The monoidal autoequivalence

$$
\gamma^{*}: \mathcal{M} \rightarrow \mathcal{M}
$$


interchanges $V$ and $V^{*}$, and hence the resulting action on the fusion semiring $R_{+}(A)$ interchanges $\alpha$ and $\beta$.

Moreover, the action of $\mathbb{Z} / 2$ on $\mathcal{M}$ allows us to define the equivariantization $\mathcal{M}^{\mathbb{Z} / 2}$ consisting of objects $W \in \mathcal{M}$ equipped with isomorphisms $\gamma^{*} W \cong W$ satisfying certain compatibility conditions (we refer the reader to [25, §3] and references therein for background and details on the equivariantization construction).

With this in place, we have

Proposition 4.1 The category $\mathcal{M}^{C}$ of finite-dimensional $C$-comodules is equivalent to the equivariantization $\mathcal{M}^{\mathbb{Z} / 2}$

Proof This is simply a dual version of [25, Proposition 3.5], where the case of a cocentral extension of a group algebra is treated in the context of semisimple Hopf algebras and categories of modules rather than comodules are considered. The arguments can be repeated virtually verbatim.

It will be important for us (for reasons that will become clear below) to work with the fixed subring $R(A)^{\mathbb{Z} / 2}$, or rather the complex fixed subalgebra $R_{\mathbb{C}}(A)^{\mathbb{Z} / 2}$. The discussion above identifies the action of $\gamma \in \mathbb{Z} / 2$ on $R_{\mathbb{C}}(A)$ with the generator-interchange map on the free algebra $\mathbb{C}\langle\alpha, \beta\rangle$.

As a consequence of Proposition 4.1, we have

Corollary 4.2 The fusion semiring $R_{+}(C)$ surjects onto the invariant part $R_{+}(A)^{\mathbb{Z} / 2}$ of the fusion semiring of $A$ under the action that interchanges $\alpha, \beta \in R_{+}(A)$.

Proof Consider the forgetful functor

$$
\text { FORGET }: \mathcal{M}^{\mathbb{Z} / 2} \rightarrow \mathcal{M}
$$

that forgets the equivariant structure on an object in $\mathcal{M}^{\mathbb{Z} / 2}$. The result follows by passing to the map

$$
R_{+}(\text {FORGET }): R_{+}(C) \rightarrow R_{+}(A)
$$

induced by FORGET at the level of fusion semirings, and observing that every finite-dimensional $A$-comodule of the form

$$
\gamma^{*} W \oplus W, W \in \mathcal{M}
$$

is in the essential image of FORGET and hence (4.3) is surjective onto $R_{+}(A)^{\mathbb{Z} / 2}$.

Finally, we obtain

Corollary 4.3 The complex fusion algebra $R_{\mathbb{C}}(C)$ is not finitely generated.

Proof Indeed, Corollary 4.2 ensures that the algebra in question surjects onto

$$
R_{\mathbb{C}}(A)^{\mathbb{Z} / 2} \cong \mathbb{C}\langle\alpha, \beta\rangle^{\mathbb{Z} / 2}
$$

In turn, the latter algebra is not finitely generated by Lemma 4.4 below.

Lemma 4.4 Let $\mathbb{Z} / 2$ act on the free ring $F=\mathbb{C}\langle\alpha, \beta\rangle$ by interchanging the generators $\alpha$ and $\beta$. Then, the invariant subalgebra $\mathbb{C}\langle\alpha, \beta\rangle^{\mathbb{Z} / 2}$ is not finitely generated over $\mathbb{C}$. 
Proof For a word $w$ consisting of letters $\alpha$ and $\beta$, denote by $w^{\gamma}$ the result of applying the transformation $\alpha \leftrightarrow \beta$ to $w$ and by $w^{*}$ the sum $w+w^{\gamma}$. As a vector space, $F^{\mathbb{Z} / 2}$ has a basis consisting of all distinct elements of $F$ of the form $w^{*}$.

Note that $F$ has a grading by $\operatorname{deg}(\alpha)=\operatorname{deg}(\beta)=1$. Now, assuming by contradiction that $F^{\mathbb{Z} / 2}$ is finitely generated, we may as well suppose that the set of elements $w^{*}$ with $\operatorname{deg}(w) \leqslant k$ generates for some $k>1$. We now seek to achieve a contradiction by showing that the subalgebra generated by such $w^{*}$ cannot contain the degree- $(k+1)$ component $\left(F^{\mathbb{Z} / 2}\right)_{k+1}$.

To this aim, let $w^{*} \in F^{\mathbb{Z} / 2}$ be an arbitrary element with $\operatorname{deg}(w)=k+1$, and assume it can be written as a linear combination of products

$$
w_{1}^{*} \cdots w_{t}^{*}, \sum_{i=1}^{t} \operatorname{deg}\left(w_{i}\right)=k+1, \operatorname{deg}\left(w_{i}\right)>0 .
$$

Note furthermore that a product (4.4) is contained in the span of products

$$
w_{1}^{*} w_{2}^{*}, \operatorname{deg}\left(w_{1}\right)+\operatorname{deg}\left(w_{2}\right)=k+1, \operatorname{deg}\left(w_{i}\right)>0,
$$

so we may as well assume we are only dealing with these latter ones (i.e. $t=2$ in all terms (4.4) in the decomposition of $\left.w^{*}\right)$.

We construct a graph having

$$
V_{k+1}=\left\{w^{*} \mid \operatorname{deg}(w)=k+1\right\}
$$

as vertices by placing an edge between $w^{*}$ and $v^{*}$ whenever

$$
w_{1}^{*} w_{2}^{*}=w^{*}+v^{*}
$$

for some $w_{i}$ as in (4.5). The graph $\Gamma_{k+1}$ in question is (isomorphic to) the hypercube with $2^{k}$ vertices, and a linear combination of elements $w^{*}$ for $\operatorname{deg}(w)=k+1$ can be regarded as a linear combination of vertices of this hypercube. Now, $\Gamma_{k+1}$ is bipartite and we will henceforth refer to the vertices in the two parts as black and white respectively. Each product (4.5) assigns equal coefficients to two vertices of opposite colors and coefficient zero to every other vertex. In other words, the linear invariant

$$
\text { (sum of coefficients of black vertices) - (sum of coefficients of white vertices) }
$$

vanishes for linear combinations of products of the form (4.5). On the other hand, a single element $w^{*}, \operatorname{deg}(w)=k+1$, when regarded as a linear combination of vertices of $\Gamma_{k+1}$, assigns coefficient 1 to a single vertex and zero to all other vertices. In conclusion, its invariant (4.6) is \pm 1 , contradicting the assumption that $w^{*}$ can be written as a linear combination of (4.5).

Finally, we can phrase the conclusion of the present section as follows:

Corollary 4.5 There exist compact quantum groups whose underlying CQG algebra is finitely presented and whose complexified fusion algebra is not finitely generated.

Proof $C$ is such an object: as an algebra it is simply the tensor product $C(\mathbb{Z} / 2) \otimes A$, and is hence finitely presented because $A$ is. On the other hand, the non-finite generation of the fusion algebra is Corollary 4.3 above. 


\section{References}

[1] N. Andruskiewitsch and J. Devoto. Extensions of Hopf algebras. Algebra i Analiz, 7(1):22-61, 1995.

[2] D. Avitzour. Noncommutative topological dynamics. ii. Trans. Amer. Math. Soc., 282(1):121$135,1982$.

[3] Teodor Banica. Le groupe quantique compact libre U(n). Comm. Math. Phys., 190(1):143-172, 1997.

[4] Teodor Banica. Quantum automorphism groups of small metric spaces. Pacific J. Math., 219(1):27-51, 2005.

[5] George M. Bergman and Saharon Shelah. Closed subgroups of the infinite symmetric group. Algebra Universalis, 55(2-3):137-173, 2006. Special issue dedicated to Walter Taylor.

[6] Jyotishman Bhowmick and Debashish Goswami. Quantum group of orientation-preserving Riemannian isometries. J. Funct. Anal., 257(8):2530-2572, 2009.

[7] Jyotishman Bhowmick, Adam Skalski, and Piotr M. Soł tan. Quantum group of automorphisms of a finite quantum group. J. Algebra, 423:514-537, 2015.

[8] Julien Bichon. Quantum automorphism groups of finite graphs. Proc. Amer. Math. Soc., 131(3):665-673 (electronic), 2003.

[9] Mathijs S. Dijkhuizen and Tom H. Koornwinder. CQG algebras: a direct algebraic approach to compact quantum groups. Lett. Math. Phys., 32(4):315-330, 1994.

[10] P. Fima, K. Mukherjee, and I. Patri. On compact bicrossed products. arXiv:1504.00092, March 2015.

[11] D. Goswami. Existence of quantum isometry group for a class of compact metric spaces. arXiv:1205.6099, May 2012.

[12] D. Goswami and S. Joardar. Rigidity of action of compact quantum groups on compact, connected manifolds. arXiv:1309.1294, September 2013.

[13] W.L. Green. Topological dynamics and $c^{*}$-algebras. Trans. Amer. Math. Soc., 210:107-121, 1975 .

[14] Paul R. Halmos. On automorphisms of compact groups. Bull. Amer. Math. Soc., 49:619-624, 1943.

[15] David Handelman. Representation rings as invariants for compact groups and limit ratio theorems for them. Internat. J. Math., 4(1):59-88, 1993.

[16] K. Iwasawa. On some types of topological groups. Ann. of Math.(2), 50:507-558, 1949.

[17] Wojciech Jaworski. Strong approximate transitivity, polynomial growth, and spread out random walks on locally compact groups. Pacific J. Math., 170(2):517-533, 1995.

[18] Wojciech Jaworski. Contraction groups, ergodicity, and distal properties of automorphisms of compact groups. Illinois J. Math., 56(4):1023-1084, 2012. 
[19] Pawel Kasprzak, Adam Skalski, and Piotr M. Sołtan. The canonical central exact sequence for locally compact quantum groups. arXiv:1508.02943, 2016.

[20] Pawel Kasprzak, Piotr M. Soltan, and Stanisław L. Woronowicz. Quantum automorphism groups of finite quantum groups are classical. J. Geom. Phys., 89:32-37, 2015.

[21] Yitzhak Katznelson. Ergodic automorphisms of $T^{n}$ are Bernoulli shifts. Israel J. Math., 10:186-195, 1971.

[22] Bruce Kitchens and Klaus Schmidt. Automorphisms of compact groups. Ergodic Theory Dynam. Systems, 9(4):691-735, 1989.

[23] Bruce P. Kitchens. Expansive dynamics on zero-dimensional groups. Ergodic Theory Dynam. Systems, 7(2):249-261, 1987.

[24] Kunal Mukherjee and Issan Patri. Automorphisms of compact quantum groups. Submitted, 2016 .

[25] Sonia Natale. Hopf algebra extensions of group algebras and Tambara-Yamagami categories. Algebr. Represent. Theory, 13(6):673-691, 2010.

[26] Issan Patri. Normal subgroups, center and inner automorphisms of compact quantum groups. Internat. J. Math., 24(9):1350071, 37, 2013.

[27] Federico Rodriguez Hertz. Stable ergodicity of certain linear automorphisms of the torus. Ann. of Math. (2), 162(1):65-107, 2005.

[28] Graeme Segal. The representation ring of a compact lie group. Inst. Hautes tudes Sci. Publ. Math., 34(1):113-128, 1968.

[29] Alfons Van Daele and Shuzhou Wang. Universal quantum groups. Internat. J. Math., 7(2):255263, 1996.

[30] Shuzhou Wang. Free products of compact quantum groups. Comm. Math. Phys., 167(3):671692, 1995.

[31] Shuzhou Wang. Tensor products and crossed products of compact quantum groups. Proc. London Math. Soc. (3), 71(3):695-720, 1995.

[32] Shuzhou Wang. Quantum symmetry groups of finite spaces. Comm. Math. Phys., 195(1):195211, 1998.

[33] S. L. Woronowicz. Compact matrix pseudogroups. Comm. Math. Phys., 111(4):613-665, 1987.

[34] S. L. Woronowicz. Compact quantum groups. In Symétries quantiques (Les Houches, 1995), pages 845-884. North-Holland, Amsterdam, 1998.

[35] Manoj K. Yadav. Class preserving automorphisms of finite p-groups: a survey. In Groups St Andrews 2009 in Bath. Volume 2, volume 388 of London Math. Soc. Lecture Note Ser., pages 569-579. Cambridge Univ. Press, Cambridge, 2011.

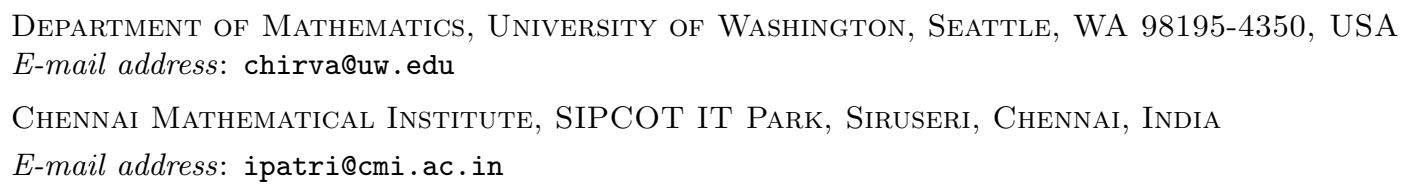

